\title{
ESSENTIAL SPECTRUM FOR A HILBERT SPACE OPERATOR
}

\author{
BY \\ RICHARD BOULDIN
}

\begin{abstract}
Various notions of essential spectrum have been defined for densely defined closed operators on a Banach space. This paper shows that the theory for those notions of essential spectrum simplifies if the underlying space is a Hilbert space and the operator is reduced by its finite-dimensional eigenspaces. In that situation this paper classifies each essential spectrum in terms of the usual language for the spectrum of a Hilbert space operator. As an application this paper deduces the main results of several recent papers dealing with generalizations of the Weyl theorem.
\end{abstract}

1. Introduction. The purpose of this paper is to show that the theory of essential spectrum for Hilbert space operators is much simpler than has been suggested by the literature on the subject. For a Hilbert space operator reduced by its finitedimensional geometric eigenspaces we show that most of the popular notions of essential spectrum coincide and we classify this set using the standard terminology for the spectrum of a Hilbert space operator. We clarify the contrast between algebraic multiplicity and geometric multiplicity for an isolated eigenvalue and thereby we obtain most of the results of the recent papers [1], [2], [5], and [10] which consider the essential spectrum of a Hilbert space operator. In the final section we list some of the applications of these results. Theorem 5 and Theorem 8 generalize the classical theorem of Weyl for essential spectrum.

2. Preliminaries. Throughout this paper we shall use "operator" to mean a linear operator defined on a vector space which is dense in the fixed underlying Hilbert space $H$. If $T$ is such an operator and $H_{0}$ is a subspace of $H$ invariant under $T$ then $T / H_{0}$ denotes the restriction of $T$ to $H_{0}$. We write the scalar operator $z I$ as simply $z$ when it causes no confusion and $z^{*}$ denotes the complex conjugate of $z$ or equivalently the adjoint of the scalar operator. By $W(T)$ we mean the numerical range of the operator $T$, i.e. $W(T)=\{\langle T f, f\rangle: f \in H,\|f\|=1\}$, and the closure of any set $S$ is written $S^{-}$. We say $\lambda$ is an isolated point of $\sigma(T)$ to mean there is no sequence $\left\{\lambda_{n}: \lambda_{n} \neq \lambda, \lambda_{n} \in \sigma(T)\right\}$ which converges to $\lambda$.

An operator $T$ is said to be Fredholm if dimension [kernel $T$ ] $<\infty, T H$ is closed, and codimension $T H<\infty$. If $T$ is Fredholm then the index of $T$ is

$$
\text { [dim } \operatorname{ker} T-\operatorname{codim} T H] \text { ]. }
$$

Received by the editors June 4, 1970.

AMS 1969 subject classifications. Primary 4615, 4710; Secondary 4748, 4730.

Key words and phrases. Essential spectrum, operator on a Hilbert space, eigenvalue, algebraic multiplicity, geometric multiplicity, Fredholm operator, index, closed range.

Copyright (c) 1972, American Mathematical Society 
We recall the following definitions of essential spectrum which have been given for closed operators in a Banach space. A point $\lambda$ from the spectrum of $T$, denoted $\sigma(T)$, is in the essential spectrum of Wolf, denoted $\sigma_{e 1}(T)$, provided that either $(T-\lambda) H$ is not closed or else it has infinite codimension. The essential spectrum of Fredholm, denoted $\sigma_{e 2}(T)$, is $\{\lambda \in \sigma(T):(T-\lambda)$ is not Fredholm $\}$; the essential spectrum of Weyl, denoted $\sigma_{e 3}(T)$, is $\{\lambda \in \sigma(T):(T-\lambda)$ is not a Fredholm operator with index equal to 0$\}$; the essential spectrum of Browder, denoted $\sigma_{e 4}(T)$, is $\{\lambda \in \sigma(T)$ : it is not the case that $(T-\lambda)$ is Fredholm with index equal to 0 and $\lambda$ is an isolated point of $\sigma(T)\}$. It is obvious that $\sigma_{e 1}(T) \subset \sigma_{e 2}(T) \subset \sigma_{e 3}(T) \subset \sigma_{e 4}(T)$. In general, in a Banach space these sets are known to be distinct.

The following lemma which is well known will be crucial to the theory developed in this paper.

LEMMA 1. Let $T$ be a closed operator on $H$ and let $\lambda$ be an isolated point of $\sigma(T)$. Then there is a direct sum decomposition of $H$, say $H_{1} \oplus A(\lambda)$, such that $H_{1}$ and $A(\lambda)$ are each invariant under $(T-\lambda)$; let $S=(T-\lambda) / H_{1}$ and $N=(T-\lambda) / A(\lambda)$. Furthermore $S$ is one-to-one and onto and $N$ is a bounded quasinilpotent operator. There is a bounded idempotent operator $P$ such that $P H=A(\lambda)$ and $(I-P) H=H_{1}$.

There are many sources for this information; we have used pp. 178-181 of [6]. The conclusions about $S$ follow from the fact that $\lambda \notin \sigma(S)$ and the conclusions about $N$ follow from the fact that $\sigma(N)=\{\lambda\}$. We note that any finite-dimensional quasinilpotent operator is, in fact, nilpotent.

Using the notation of the lemma we shall refer to $A(\lambda)$ as the spectral subspace associated with $\lambda$ and $\operatorname{dim} A(\lambda)$ is the algebraic multiplicity of $\lambda$. We define $G(\lambda)$ to be $\operatorname{ker}(T-\lambda)$ and we refer to this as the geometric eigenspace associated with $\lambda$ and $\operatorname{dim} G(\lambda)$ is the geometric multiplicity of $\lambda$. Of course $G(\lambda)$ is contained in $A(\lambda)$ and in the special case that $G(\lambda)$ is nontrivial we shall refer to $A(\lambda)$ as the algebraic eigenspace associated with $\lambda$. It need not be the case that $G(\lambda)$ is nontrivial. Consider the Volterra integration operator, say $V$, and recall that $\sigma(V)=\{0\}$, although $V$ is one-to-one (see pp. 94-95 of [4]).

3. Classification of essential spectrum. In this section we shall prove some basic facts about the spectrum of Hilbert space operator; the analogous assertions for a Banach space operator are easily seen to be false.

THEOREM 1. Let $T$ be a closed operator on $H$ which is reduced by $G(\lambda)=\operatorname{ker}(T-\lambda)$ and let $\left\{\lambda_{n}\right\}$ be a sequence such that $\lambda_{n} \in \sigma(T), \lambda_{n} \neq \lambda$, and $\lambda_{n} \rightarrow \lambda$. If for each $n=1$, $2, \ldots$ and $\varepsilon>0$ there exists a unit vector $f(\varepsilon) \in H$ such that $\left\|\left(T-\lambda_{n}\right) f(\varepsilon)\right\|<\varepsilon$ then $(T-\lambda) H$ is not closed.

Proof. For each $n$ take a unit vector $f_{n}$ such that $\left\|\left(T-\lambda_{n}\right) f_{n}\right\|<\left|\lambda-\lambda_{n}\right|^{2}$. Decompose $f_{n}$ into $f_{n}^{\prime}+f_{n}^{\prime \prime}$ where $f_{n}^{\prime} \in G(\lambda)$ and $f_{n}^{\prime \prime} \in[G(\lambda)]^{\perp}$ and note that $\left(T-\lambda_{n}\right) f_{n}^{\prime}=$ $(T-\lambda) f_{n}^{\prime}+\left(\lambda-\lambda_{n}\right) f_{n}^{\prime}=\left(\lambda-\lambda_{n}\right) f_{n}^{\prime}$. Because $G(\lambda)$ reduces $\left(T-\lambda_{n}\right)$ we have

$$
\begin{aligned}
\left\|\left(T-\lambda_{n}\right) f_{n}\right\|^{2} & =\left\|\left(T-\lambda_{n}\right) f_{n}^{\prime}\right\|^{2}+\left\|\left(T-\lambda_{n}\right) f_{n}^{\prime \prime}\right\|^{2} \\
& =\left|\lambda-\lambda_{n}\right|^{2}\left\|f_{n}^{\prime}\right\|^{2}+\left\|\left(T-\lambda_{n}\right) f_{n}^{\prime \prime}\right\|^{2} .
\end{aligned}
$$


Thus, in particular, $\left|\lambda-\lambda_{n}\right|^{4}>\left|\lambda-\lambda_{n}\right|^{2}\left\|f_{n}^{\prime}\right\|^{2}$ and consequently $\left\|f_{n}^{\prime}\right\| \rightarrow 0$ and $\left\|f_{n}^{\prime \prime}\right\|^{2}$ $=\left(1-\left\|f_{n}^{\prime}\right\|^{2}\right) \rightarrow 1$. Hence we may assume that $\left\|f_{n}^{\prime \prime}\right\| \geqq \frac{1}{2}$ and it follows from $(+)$ above that $\left\|\left(T-\lambda_{n}\right) f_{n}^{\prime \prime}\right\| \rightarrow 0$. So we may replace the original sequence $\left\{f_{n}\right\}$ with $\left\{f_{n}^{\prime \prime} /\left\|f_{n}^{\prime \prime}\right\|\right\}$ or equivalently we shall assume that $f_{n} \in[G(\lambda)]^{\perp}$.

It follows that $(T-\lambda)^{+}(T-\lambda) f_{n}=f_{n}$ where $(T-\lambda)^{+}$is the linear transformation inverse to $(T-\lambda):[G(\lambda)]^{\perp} \rightarrow[(T-\lambda) H]^{-}$. If $(T-\lambda) H$ is closed then $(T-\lambda)^{+}$is bounded by application of the closed graph theorem, noting that $(T-\lambda) /[G(\lambda)]^{\perp}$ is a closed operator. By the triangle inequality we see that $\left\|(T-\lambda) f_{n}\right\| \rightarrow 0$ and consequently if $(T-\lambda) H$ is closed we have $\left\|(T-\lambda)^{+}(T-\lambda) f_{n}\right\| \rightarrow 0$. The fact that $\left\|(T-\lambda)^{+}(T-\lambda) f_{n}\right\|=\left\|f_{n}\right\|=1$ shows that $(T-\lambda) H$ is not closed.

Corollary 1. Let $T$ be a closed operator on $H$ which is reduced by $G^{*}\left(\lambda^{*}\right)$ $=\operatorname{ker}\left(T^{*}-\lambda^{*}\right)$ and let $\left\{\lambda_{n}\right\}$ be a sequence such that $\lambda_{n} \in \sigma(T), \lambda_{n} \neq \lambda$, and $\lambda_{n} \rightarrow \lambda$. If for each $n$ the operator $\left(T-\lambda_{n}\right)$ is one-to-one with closed range then $(T-\lambda) H$ is not closed.

Proof. Because $\lambda_{n} \in \sigma(T)$ it must be that $\left[\left(T-\lambda_{n}\right) H\right]^{\perp} \neq\{0\}$; otherwise we could use the closed graph theorem as in the preceding proof to conclude that $\left(T-\lambda_{n}\right)^{-1}$ is everywhere defined and bounded. Since $\operatorname{ker}\left(T^{*}-\lambda_{n}^{*}\right)=\left[\left(T-\lambda_{n}\right) H\right]^{\perp}$ we see that for each $\varepsilon>0$ there exists a unit vector $f(\varepsilon)$ such that $\left\|\left(T^{*}-\lambda_{n}^{*}\right) f(\varepsilon)\right\|=0<\varepsilon$. We apply the preceding theorem to $T^{*},\left\{\lambda_{n}^{*}\right\}$, and $\lambda^{*}$ and thus we conclude that $\left(T^{*}-\lambda^{*}\right) H$ is not closed. Then $(T-\lambda) H$ is not closed according to the closed range theorem (see pp. 205-208 of [14]).

In the situation dealt with in the preceding corollary we can reach the weaker conclusion that $(T-\lambda)$ is not a Fredholm operator with a weaker hypothesis by using well-known theory for the stability of the index.

LEMMA 2. Let $T$ be a closed operator and let $\left\{\lambda_{n}\right\}$ be a sequence such that $\lambda_{n} \in \sigma(T)$, $\lambda_{n} \neq \lambda$, and $\lambda_{n} \rightarrow \lambda$. If for each $n$ the operator $\left(T-\lambda_{n}\right)$ is one-to-one with closed range then $(T-\lambda)$ is not a Fredholm operator.

Proof. Because $\left(T-\lambda_{n}\right)$ converges to $(T-\lambda)$ in the operator norm we would contradict Theorem 5.17, p. 235, of [6] if $(T-\lambda)$ were a Fredholm operator.

We are now able to complete our consideration of nonisolated points of the spectrum.

THEOREM 2. Let $T$ be a closed operator which is reduced by the subspace $G(\lambda)$ $=\operatorname{ker}(T-\lambda)$ and let $\left\{\lambda_{n}\right\}$ be a sequence such that $\lambda_{n} \in \sigma(T), \lambda_{n} \neq \lambda$, and $\lambda_{n} \rightarrow \lambda$. Then $(T-\lambda)$ is not a Fredholm operator. Furthermore, if $G^{*}\left(\lambda^{*}\right)=\operatorname{ker}\left(T^{*}-\lambda^{*}\right)$ reduces $T$ then $(T-\lambda) H$ is not closed.

Proof. For $\lambda_{n} \in \sigma(T)$ there are only three possibilities: (a) $\left(T-\lambda_{n}\right)$ is not one-toone, (b) $\left(T-\lambda_{n}\right)$ is one-to-one but $\left(T-\lambda_{n}\right) H$ is not closed, (c) $\left(T-\lambda_{n}\right)$ is one-to-one with closed range but $\left[\left(T-\lambda_{n}\right) H\right]^{\perp} \neq\{0\}$. Otherwise apply the closed graph theorem to see that $\left(T-\lambda_{n}\right)^{-1}$ is everywhere defined and bounded. 
Assuming (a) it is obvious that for any $\varepsilon>0$ there exists a unit vector $f(\varepsilon)$ such that $\left\|\left(T-\lambda_{n}\right) f(\varepsilon)\right\|=0<\varepsilon$. Assuming (b) it follows from the well-known theory of the reduced minimum modulus (see pp. 231-232 of [6]) that for any $\varepsilon>0$ there exists a unit vector $f(\varepsilon)$ with $\left\|\left(T-\lambda_{n}\right) f(\varepsilon)\right\|<\varepsilon$.

Now let $\left\{\lambda_{n}\right\}$ be any sequence such that $\lambda_{n} \in \sigma(T), \lambda_{n} \neq \lambda$, and $\lambda_{n} \rightarrow \lambda$. Either there is an infinite subset of $\left\{\lambda_{n}\right\}$ satisfying one of the conditions (a), (b), in which case we apply Theorem 1, or else there is an infinite subset of $\lambda_{n}$ satisfying (c) in which case we apply Lemma 2 . In this latter case if we have assumed that $G^{*}\left(\lambda^{*}\right)$ reduces $T$ then we may apply Corollary 1 to conclude that $(T-\lambda) H$ is not closed.

The one remaining tool that we need for this section is the next lemma which we shall deduce from some theorems in [6]. As Kato mentions, the method of proof for the main theorem cited is the same method used by A. E. Taylor, Dunford and Schwartz, and others.

Lemma 3. Let $T$ be a bounded quasinilpotent operator on $H$. If $\operatorname{dim} H=\infty$ then either $T H$ is not closed or else $\operatorname{dim} \operatorname{ker} T=\operatorname{codim} T H=\infty$.

Proof. If $T H$ is not closed there is nothing to prove; so we may assume that $T H$ is closed. By Theorem 5.30, p. 240, of [6] we have that nul' $T=\operatorname{def}^{\prime} T=\infty$. According to Theorem 5.10 , p. 233 , of the same source, nul' $T \geqq \operatorname{dim} \operatorname{ker} T$ and $\operatorname{def}^{\prime} T$ $\geqq \operatorname{codim} T H$ with the equalities holding if $T H$ is closed. Hence we have that $\operatorname{dim} \operatorname{ker} T=\operatorname{codim} T H=\infty$ as desired.

We come to the main result of this section.

THEOREM 3. If $T$ is a closed operator which is reduced by each finite-dimensional subspace $\operatorname{ker}(T-z)$ for any complex number $z$ then (2), (3), (4), and (5), from the following sets, are identical. Furthermore if $T$ is also reduced by each finite-dimensional subspace $\operatorname{ker}\left(T^{*}-z\right)$ then all five sets are identical:

(1) $\sigma_{e 1}(T)$,

(2) $\sigma_{e 2}(T)$,

(3) $\sigma_{e 3}(T)$,

(4) $\sigma_{e 4}(T)$,

(5) the points of $\sigma(T)$ which are not isolated eigenvalues of finite algebraic multiplicity.

Proof. By Theorem 2 if $\lambda$ is not an isolated point of $\sigma(T)$ then $(T-\lambda)$ is not a Fredholm operator and it follows that $\lambda$ is in each of the above sets (2), (3), (4), (5). If we assume the further hypothesis that each finite-dimensional $\operatorname{ker}\left(T^{*}-z\right)$ reduces $T$ then either $(T-\lambda) H$ is not closed or else $\operatorname{codim}(T-\lambda) H=\infty$, and $\lambda$ is in each of the above five sets.

Thus we may assume that $\lambda$ is an isolated point of $\sigma(T)$ and we consider two cases according to whether $A(\lambda)$ is infinite dimensional or finite dimensional. First we assume that $A(\lambda)$ is finite dimensional and we apply the facts and notation of Lemma 1. Because $(T-\lambda)=S \oplus N$ we have $\operatorname{dim} \operatorname{ker}(T-\lambda)=\operatorname{dim} \operatorname{ker} S+\operatorname{dim} \operatorname{ker} N$ 
and since $S$ is one-to-one and onto this simplifies to $\operatorname{dim} \operatorname{ker}(T-\lambda)=\operatorname{dim} \operatorname{ker} N<\infty$. Similarly, codim $(T-\lambda) H=\operatorname{codim} N A(\lambda)<\infty$. Using that $A(\lambda)$ is finite dimensional and a standard isomorphism theorem for finite-dimensional vector spaces we conclude that $N A(\lambda)$ is closed with $\operatorname{dim} \operatorname{ker} N=\operatorname{codim} N A(\lambda)$. Thus $(T-\lambda)$ is a Fredholm operator with index zero and $\lambda$ is isolated from $\sigma(T)$. It is clear that any nilpotent operator must have nontrivial kernel and so $\lambda$ is an eigenvalue for $T$. Hence $\lambda$ is not in any of the sets (1), (2), (3), (4), or (5).

Finally we consider that $\lambda$ is an isolated point of $\sigma(T)$ with $\operatorname{dim} A(\lambda)=\infty$. By Lemma $1, N$ is a bounded quasinilpotent operator and so we can conclude from Lemma 3 that either $N A(\lambda)$ is not closed (and consequently $(T-\lambda) H=H_{1} \oplus N A(\lambda)$ is not closed) or else codim $N A(\lambda)=\infty$ (and consequently codim $(T-\lambda) H=\infty$ ). Thus $\lambda$ is in all of the sets (1), (2), (3), (4), and (5). Since we have considered all possibilities for $\lambda \in \sigma(T)$ the theorem follows.

COROllary 2. Let $T$ be a closed operator which is reduced by each finite-dimensional subspace $\operatorname{ker}(T-z)$ or $\operatorname{ker}\left(T^{*}-z\right)$, for any complex number $z$. Then $\lambda$ is an isolated eigenvalue of finite algebraic multiplicity for $T$ if and only if $\lambda^{*}$ is the same for $T^{*}$.

Proof. It is well known that $(T-\lambda)$ is Fredholm with index zero if and only if $\left(T^{*}-\lambda^{*}\right)$ is Fredholm with index zero. Now use that (3) and (5) are the same set for both $T$ and $T^{*}$.

4. Geometric multiplicity versus algebraic multiplicity. The papers [7], [3], [12] proved remarkable theorems about the stability of the index of $(T-\lambda)$ as $\lambda$ varies and then these stability theorems were used to deduce a theory for the invariance of essential spectrum as defined by several different authors. The more recent papers [2], [5], [1], [10] have extended the classical essential spectrum theorem of Weyl for normal operators to larger classes of Hilbert space operators by interpreting the Banach space definition of the Weyl spectrum (as given in §2) for an operator in one of these larger classes; these authors have shown that for such an operator the Weyl spectrum is the set of points in the spectrum which are not isolated eigenvalues of finite geometric multiplicity. We consider our Theorem 3 to be a strong argument that the natural generalization of Weyl's theorem (see $\S 5$ Theorem 5) uses algebraic multiplicity rather than geometric multiplicity.

In this section we shall deduce a number of conditions for the two multiplicities to coincide for an isolated eigenvalue. In Theorem 4, which summarizes these results, condition (3) with our Theorem 3 gives a notable improvement on the result of Berberian in [1] and, since he deduced Istrătescu's result, our (3) includes that theorem also. Our condition has the advantage not only of being properly weaker but also simpler. Similarly (1), in view of Theorem 3, contains Coburn's main result in [2] and (2) contains the result of Nieto [10] in the case that the underlying Banach space is a Hilbert space. Part (4) has no antecedent known to us. The 
effect of Theorem 8 of $\S 5$, which applies Theorem 4 , is stronger than any of the previously mentioned results because different conditions can be applied to different isolated points of the spectrum.

Recall that a bounded operator $T$ is seminormal provided that $T^{*} T-T T^{*}$ is either nonnegative or nonpositive and in the former case $T$ is said to be hyponormal. If $T$ is a bounded operator in the Hilbert space $(\langle\cdot, \cdot\rangle, H)$ and there exists a new inner product, say $\langle\langle\cdot, \cdot\rangle\rangle$, such that $T$ is symmetric with respect to it and $\langle\langle f, f\rangle \leqq c\langle f, f\rangle$ for some constant $c$ and all $f \in H$ then $T$ is said to be symmetrizable. Most of the following theorem can be extended to the case that $T$ is a closed operator. This would require generalizing the basic theory for the above operators and such a process involves tedious considerations of domain. Therefore we state and prove the theorem only for a bounded operator $T$.

THEOREM 4. Let $\lambda$ be an isolated point of $\sigma(T)$ for the bounded operator $T$. If $T$ satisfies any one of the following conditions then the spectral subspace, $A(\lambda)$, coincides with the geometric eigenspace, $G(\lambda)$ :

(1) $H$ can be written as the direct sum of subspaces $H_{1}, H_{2}$ each of which is invariant under $T$ with $T / H_{1}$ hyponormal and $\lambda \notin \sigma\left(T / H_{2}\right)$,

(2) instead of $T / H_{1}$ being hyponormal it is symmetrizable,

(3) $H_{0}=[G(\lambda)]^{\perp}$ is invariant under $T$ and $\lambda \notin \sigma\left(T / H_{0}\right)$,

(4) for any half line originating at $\lambda$, call it $L$, we have $\left\|(T-z)^{-1}|A(\lambda) \| \leqq| z-\left.\lambda\right|^{-1}\right.$ for $z \in L$ and $|z|$ sufficiently large.

Proof of (1). Since $\lambda \notin \sigma\left(T / H_{2}\right)$ we may assume that $H_{1}=H$. A simple computation shows that $(T-\lambda)$ is hyponormal because $T$ is hyponormal. Since $A(\lambda)$ is invariant under $(T-\lambda)$ and the adjoint of the restriction is $P\left(T^{*}-\lambda^{*}\right)$ where $P$ is the orthogonal projection onto $A(\lambda)$, it follows that $(T-\lambda) / A(\lambda)$ is hyponormal. For any hyponormal operator the spectral radius equals the norm (see Problem 162 of [4]) and thus the quasinilpotent operator $(T-\lambda) / A(\lambda)$ is zero.

Proof of (2). As in (1) we may assume $H=H_{1}$. Let $(\langle\cdot, \cdot\rangle, H)$ be the usual underlying Hilbert space and let $\left(\left\langle\langle\cdot, \cdot\rangle, H^{\prime}\right)\right.$ be the Hilbert space formed by completing $H$ with respect to the norm arising from $\langle\langle\cdot, \cdot\rangle\rangle$ where $T$ is symmetric with respect to $\left\langle\langle\cdot, \cdot\rangle\right.$. Because $\sigma\left(T / H^{\prime}\right) \subset \sigma(T / H)$ (see Theorem II of [8]) if $\lambda \in \sigma\left(T / H^{\prime}\right)$ is an isolated point of $\sigma(T / H)$ it is the same for $\sigma\left(T / H^{\prime}\right)$. Let $P_{1}$ be the orthogonal projection onto $\operatorname{ker}(T-\lambda) / H^{\prime}$, which is also the spectral subspace associated with $\lambda$ since $T / H^{\prime}$ is selfadjoint. The essential observation, which also appeared in Nieto's work, is that

$$
P_{1} / H=-(1 / 2 \pi i) \int_{|z-\lambda|=\varepsilon}[(T-z) / H]^{-1} d z
$$

and the integral defines the idempotent onto the spectral subspace for $T / H$ corresponding to $\lambda$. Since $(T-\lambda) P_{1} / H^{\prime}=0$ it is clear that $(T-\lambda) P_{1} / H=0$ and so the spectral subspace for $T / H$ corresponding to $\lambda$ coincides with the geometric eigenspace. 
Proof of (3). Let $S$ be $(T-\lambda) / H_{0}$ and note that $S$ has a bounded everywhere defined inverse. Thus inf $\{\|S f\| /\|f\|: f \neq 0\}=\inf \{\|S f\|:\|f\|=1\}=1 /\left\|S^{-1}\right\|>0$ and an easy induction argument shows that $\inf \left\{\left\|S^{n} f\right\|:\|f\|=1\right\} \geqq 1 /\left\|S^{-1}\right\|^{n}$. Now $H_{1}=A(\lambda) \cap[G(\lambda)]^{\perp}$ is invariant under $T$, and thus under $T-\lambda$. Then $S / H_{1}$ is quasinilpotent although $\left\|S^{n} / H_{1}\right\| \geqq 1 /\left\|S^{-1}\right\|^{n}$ by the above inequality. Because $\left\|S^{n} / H_{1}\right\|^{1 / n} \geqq 1 /\left\|S^{-1}\right\|$ we know that the spectral radius of $S / H_{1}$ is strictly positive and this contradicts that $S / H_{1}$ is quasinilpotent unless $H_{1}=\{0\}$.

Proof of (4). We know that $A(\lambda)$ is invariant under $T$, for $z \notin \sigma(T)$ the resolvent $[T / A(\lambda)-z]^{-1}$ is $[T-z]^{-1} / A(\lambda)$ and $\sigma(T / A(\lambda))=\{\lambda\}$. By examining the proof of Orland's result [11] we see that if $\left\|[T / A(\lambda)-z]^{-1}\right\| \leqq|z-\lambda|^{-1}$ for $z \in L$ and $|z|$ sufficiently large then $W(T / A(\lambda))^{-}$is the closed convex hull of $\sigma(T / A(\lambda))$. For $|z|$ sufficiently large we have that $z \neq \lambda$ and $z \notin \sigma(T)$. Hence our hypothesis is sufficient to conclude that $W(T / A(\lambda))^{-}=\{\lambda\}$; it is well known that this suffices for $T / A(\lambda)=\lambda$ and so $A(\lambda)=G(\lambda)$.

In order to replace the phrase "finite algebraic multiplicity" in Theorem 3 with the phrase "finite geometric multiplicity" we do not have to show that $A(\lambda)$ and $G(\lambda)$ coincide whenever $\lambda$ is an isolated point of $\sigma(T)$. We do have to show that if $\lambda$ is an isolated point of $\sigma(T)$ and $G(\lambda)$ is finite dimensional then $A(\lambda)$ is finite dimensional.

COROLlaRy 3. Let $T$ be a bounded operator which is reduced by each finitedimensional subspace $\operatorname{ker}(T-z)$ for any complex number $z$. If for each isolated point of $\sigma(T)$, say $\lambda$, either one of the properties (1), (2), (3), or (4) of Theorem 4 holds or else (5) below holds, then the Weyl essential spectrum of $T$ consists of the points of $\sigma(T)$ which are not isolated eigenvalues with finite geometric multiplicity:

(5) $(T-\lambda) / A(\lambda)$ is nilpotent.

Proof. Let $n$ be the index of nilpotency for $(T-\lambda) / A(\lambda)$ and note that $\operatorname{dim} A(\lambda)$ $=\operatorname{dim} \operatorname{ker}(T-\lambda)^{n} / A(\lambda) \leqq n \operatorname{dim} G(\lambda)$. So $\lambda$ has finite geometric multiplicity if and only if it has finite algebraic multiplicity. This corollary now follows from Theorem 4 and Theorem 3.

5. Applications. With the theorems which we have now proved we are able to quickly write down a number of applications. The operator $B$ is said to be $A$ compact provided that $\left\{B f_{n}\right\}$ has a convergent subsequence whenever $\left\{f_{n}\right\}$ is contained in the domain of the closed operator $A$ and $\left\{\left\|f_{n}\right\|+\left\|A f_{n}\right\|\right\}$ is bounded. Note that if $B$ is compact then $B$ is $A$-compact and $B$ is a closed operator.

THEOREM 5. Let $A$ be a closed operator which is reduced by each of its finitedimensional geometric eigenspaces and let $B$ be a closed $A$-compact operator. $A$ point $\lambda$ is in the Weyl essential spectrum of $A+B$, i.e. $\lambda \in \sigma_{e 3}(A+B)$, if and only if $\lambda \in \sigma(A)$ and $\lambda$ is not an isolated eigenvalue with finite algebraic multiplicity. Any $\lambda \in \sigma_{e 3}(A+B)$ is either a nonisolated point of $\sigma(A+B)$ or else an isolated point with infinite algebraic multiplicity. 
Proof. The characterization of $\sigma_{e 3}(A+B)$ follows from the equality of sets (3) and (5) in our Theorem 3 and from Theorem 2.1 of Schechter in [12]. It follows from the second paragraph of the proof of Theorem 3 that if $\lambda \in \sigma_{e 3}(A+B)$ then $\lambda$ is not an isolated point with finite algebraic multiplicity.

We say that the operator $B$ is $A$-pseudo-compact provided that $\left\{B f_{n}\right\}$ has a convergent subsequence whenever $\left\{f_{n}\right\}$ is contained in the domain of the closed operator $A$ and $\left\{\left\|f_{n}\right\|+\left\|A f_{n}\right\|+\left\|B f_{n}\right\|\right\}$ is bounded.

THEOREM 6. The conclusion of Theorem 5 holds provided that B is a closed A-pseudocompact operator and there are points $\eta$ and $\mu$ such that $(A-\eta)$ and $(B-\mu)$ are Fredholm operators.

Proof. This follows from the equality of sets (3) and (5) in our Theorem 3 and Schechter's Theorem 2.3 in [12].

We say that the operator $B$ is $A^{2}$-pseudo-compact provided that $\left\{B f_{n}\right\}$ has a convergent subsequence whenever $\left\{f_{n}\right\}$ is contained in the domain of the closed operator $A^{2}$ and $\left\{\left\|f_{n}\right\|+\left\|A f_{n}\right\|+\left\|B f_{n}\right\|+\left\|A^{2} f_{n}\right\|+\left\|B A f_{n}\right\|\right\}$ is bounded.

THEOREM 7. The conclusion of Theorem 5 holds provided that $B$ is a closed $A^{2}$ pseudo-compact operator and there is a point $\mu$ such that $(A-\mu)$ and $(B-\mu)$ are both Fredholm operators.

Proof. Use the equality of sets (3) and (5) in our Theorem 3 and Schechter's Theorem 2.5 in [12].

There are other similar applications that are arrived at by transcribing essential spectrum theorems according to our Theorem 3. In particular with the hypothesis of any one of the Theorems 5,6 , or 7 we can conclude that the essential spectrum of Browder for $A+B$, i.e. $\sigma_{e 4}(A+B)$, contains $\sigma_{e 4}(A)$.

From Corollary 3 we get a generalization of the classical essential spectrum theorem of Weyl and this generalization contains the main results of [2], [1], [5], and [10].

THEOREM 8. Let $A$ be a bounded operator reduced by each finite-dimensional subspace $\operatorname{ker}(A-z)$. If for each isolated point of $\sigma(A)$ one of the conditions (1), (2), (3), (4) or (5) mentioned in Corollary 3 is satisfied then the largest subset of $\sigma(A)$ which is invariant under all compact perturbations is the set of $\lambda \in \sigma(A)$ such that $\lambda$ is not an isolated eigenvalue of finite geometric multiplicity.

Proof. Use our Corollary 3 and the fact from [13] that the essential spectrum of Weyl is the largest subset of $\sigma(A)$ which is invariant under all compact perturbations.

The final application is based on an observation of Coburn in [2]. We write $w(A)$ and $r(A)$ for the numerical radius and the spectral radius, respectively, i.e. $w(A)$ $=\sup \{|z|: z \in W(A)\}$ and $r(A)=\sup \{|z|: z \in \sigma(A)\}$.

THEOREM 9. Let $A$ be a closed operator which is reduced by each finite-dimensional subspace $\operatorname{ker}(A-z)$. If $A$ has no isolated eigenvalues of finite algebraic multiplicity 
then $r(A) \leqq r(A+K)$ for every closed A-compact operator $K$. Furthermore if $w(A)$ $=\|A\|$ then $\|A\| \leqq\|A+K\|$ for every closed A-compact operator $K$.

Proof. The first assertion is immediate from Theorem 5. Lumer showed in [9] that if $w(A)=\|A\|$ then $r(A)=\|A\|$ and it is always true that $r(A+K) \leqq\|A+K\|$. Thus the second assertion follows from the first one.

Remark. Our basic hypothesis has been " $T$ is reduced by each finite-dimensional subspace $\operatorname{ker}(T-z)$ for $z$ a complex number". It is possible to replace this hypothesis with a properly weaker condition which is very geometric. This improvement is not free, however, since the new condition is somewhat more complicated. An alternative development of $\$ 3$ using the weaker geometric condition will be discussed elsewhere. (See Richard Bouldin, The Weyl essential spectrum, Proc. Amer. Math. Soc. 28 (1971), 531-536.)

\section{BIBLIOGRAPHY}

1. S. K. Berberian, An extension of Weyl's theorem to a class of not necessarily normal operators, Michigan Math. J. 16 (1969), 273-279. MR 40 \#3335.

2. L. A. Coburn, Weyl's theorem for nonnormal operators, Michigan Math. J. 13 (1966), 285-288. MR 34 \#1846.

3. I. C. Gohberg and M. G. Krein, The basic propositions on defect numbers, root numbers and indices of linear operators, Uspehi Mat. Nauk 12 (1957), no. 2 (74), 43-118; English transl., Amer. Math. Soc. Transl. (2) 13 (1960), 185-264. MR 20 \#3459; MR 22 \#3984.

4. P. R. Halmos, A Hilbert space problem book, Van Nostrand, Princeton, N. J., 1967. MR 34 \#8178.

5. V. Istrătescu, Weyl's theorem for a class of operators, Rev. Roumaine Math. Pures Appl. 13 (1968), 1103-1105. MR 38 \#6381.

6. Tosio Kato, Perturbation theory for linear operators, Die Grundlehren der math. Wissenschaften, Band 132, Springer-Verlag, New York, 1966. MR 34 \#3324.

7. - Perturbation theory for nullity, deficiency and other quantities of linear operators, J. Analyse Math. 6 (1958), 261-322. MR 21 \#6541.

8. P. D. Lax, Symmetrizable linear transformations, Comm. Pure Appl. Math. 7 (1954), 633-647. MR 16, 832.

9. G. Lumer, Semi-inner-product spaces, Trans. Amer. Math. Soc. 100 (1961), 29-43. MR 24 \#A2860.

10. Joseph Nieto, On the essential spectrum of symmetrizable operators, Math. Ann. 178 (1968), 145-153. MR 38 \#1544.

11. George Orland, On a class of operators, Proc. Amer. Math. Soc. 15 (1964), 75-79. MR 28 \#480.

12. Martin Schechter, On the essential spectrum of an arbitrary operator. I, J. Math. Anal. Appl. 13 (1966), 205-215. MR 32 \#6230.

13. - Invariance of the essential spectrum, Bull. Amer. Math. Soc. 71 (1965), 365-367. MR 30 \#5167.

14. K. Yosida, Functional analysis, Die Grundlehren der math. Wissenschaften, Band 123, Academic Press, New York; Springer-Verlag, Berlin, 1965. MR 31 \#5054.

Department of Mathematics, University of Georgia, Athens, Georgia 30601 